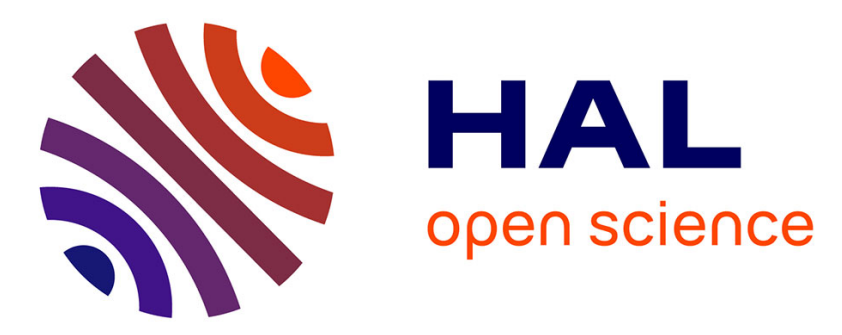

\title{
Univariate real root isolation over a single logarithmic extension of real algebraic numbers
}

Adam Strzebonski, Elias Tsigaridas

\section{To cite this version:}

Adam Strzebonski, Elias Tsigaridas. Univariate real root isolation over a single logarithmic extension of real algebraic numbers. ACA 2015 - Applications of Computer Algebra, Jul 2015, Kalamata, Greece. pp.425-445, 10.1007/978-3-319-56932-1_27. hal-01001820v3

\section{HAL Id: hal-01001820 \\ https://hal.inria.fr/hal-01001820v3}

Submitted on 24 Dec 2016

HAL is a multi-disciplinary open access archive for the deposit and dissemination of scientific research documents, whether they are published or not. The documents may come from teaching and research institutions in France or abroad, or from public or private research centers.
L'archive ouverte pluridisciplinaire HAL, est destinée au dépôt et à la diffusion de documents scientifiques de niveau recherche, publiés ou non, émanant des établissements d'enseignement et de recherche français ou étrangers, des laboratoires publics ou privés. 


\title{
Univariate real root isolation over a single logarithmic extension of real algebraic numbers
}

\author{
Adam Strzeboński* $\quad$ Elias P. Tsigaridas ${ }^{\dagger}$
}

December 5, 2016

\begin{abstract}
We present algorithmic, complexity and implementation results for the problem of isolating the real roots of a univariate polynomial $B \in L[x]$, where $L=\mathbb{Q}[\lg (\alpha)]$ and $\alpha$ is a positive real algebraic number. The algorithm approximates the coefficients of $B$ up to a sufficient accuracy and then solves the approximate polynomial. For this we derive worst case (aggregate) separation bounds. We also estimate the expected number of real roots when we draw the coefficients from a specific distribution and illustrate our results experimentally. A generalization to bivariate polynomial systems is also presented. We implemented the algorithm in $\mathrm{C}$ as part of the core library of MATHEMATICA for the case $B \in \mathbb{Z}[\lg (q)][x]$ where $q$ is positive rational number and we demonstrate its efficiency over various data sets.
\end{abstract}

\section{Introduction}

We consider the problem of isolating the real roots of a univariate polynomial the coefficients of which are polynomials in the logarithm of a positive real algebraic number. We consider two variants of the problem. In the first variant the argument of the logarithm is a positive real algebraic number. In the second the argument is a bivariate homogeneous polynomial evaluated at two real algebraic numbers. The reader can refer to the end of the introduction for a detailed presentation of the notation that we use. The first problem that we consider is the following:

Problem 1. Consider the square-free polynomial

$$
B_{\alpha}=\sum_{i=0}^{d} b_{i} x^{i}, \quad \text { where } \quad b_{i}=\sum_{j=0}^{\nu_{i}} b_{i, j}(\lg (\alpha))^{j}
$$

$b_{i, j} \in \mathbb{Z}$, the bitsize of $b_{i, j}$ is bounded by $\tau$, and $\alpha$ is a positive real root of a polynomial $A \in \mathbb{Z}[x]$ of degree $m$ and maximum coefficient bitsize $\tau$. Finally, let $\nu=\max _{i} \nu_{i}$. What is the Boolean complexity of isolating the real roots of $B_{\alpha}$ ?

The problem of isolating the real roots of a univariate polynomial is a well studied problem. However, most of the results focus on polynomials with rationals or algebraic numbers as coefficients. We are not aware of any complexity results that consider polynomials with transcendental numbers as coefficients. We present the first complexity bounds for the real solving problem for a family of polynomials with coefficients involving logarithms of algebraic numbers. In addition, our implementation is the first complete one for solving exactly polynomial with such transcendental numbers as coefficients.

\footnotetext{
${ }^{*}$ Wolfram Research Inc.,100 Trade Centre Drive, Champaign, IL 61820, U.S.A., adams@wolfram.com

${ }^{\dagger}$ POLSYS Project, INRIA Paris-Rocquencourt UPMC, Univ Paris 06, LIP6, FRANCE. elias.tsigaridas@inria.fr
} 
We tackle the problem by approximating the coefficients of $B_{\alpha}$ up to a sufficient precision. In this way we relate it to numerical univariate real solving algorithms [34, 30], see also [32, 21], and to algorithms based on the bitstream model, e.g. [26, 15, 33]. For a detailed treatment of numerical solvers we refer the reader to [25, Chapter 15]. Problem 1 is a generalization of the problem of solving polynomials with coefficients in an extension field, $[22,36,35]$, see also $[9,40,39,23]$ and references therein. We also refer to the recent work of Bates and Sottile [4] on Khovanskii-Rolle continuation algorithm that exploits logarithms of polynomial expressions. For the close related problem of computing the zeros of analytic functions using inclusion and exclusion predicates we refer the reader to [20,41, 11, 42].

To obtain the various bounds we have to combine several algebraic techniques in a novel way and to provide new evaluation and perturbation bounds; the latter turn out to be useful in other applications as well. Our analysis is based on effective lower bounds of linear forms in two logarithms; a result due to Mignotte and Waldschmidt (Thm. 3). We combine this bound with univariate and multivariate separation and evaluation bounds of polynomials and polynomial systems. The idea is to approximate the coefficients of $B_{\alpha}$ up to a sufficient precision and then isolate the real roots of the approximate polynomial. The precision is such that the number of the real roots remains the same and from the isolated intervals of the approximate polynomial we can derive isolating intervals for the real roots of $B_{\alpha}$.

First, we need to quantify "sufficient accuracy". We treat the logarithm as a parameter and the separation bound of $B_{\alpha}$ turns out to be a univariate polynomial in this parameter. We estimate a lower bound on this evaluation by proving that it depends only on the closest root and the separation bound of the polynomial (Lemma 2) and combining it with Thm. 3. This approach saves us a factor compared to the straightforward one of factoring the polynomial in linear factors and bounding the separation using Thm. 3 directly.

This approach turns out to be applicable for tackling a more general problem, Problem 2, where the argument of the logarithm is a homogeneous bivariate polynomial evaluated at two real algebraic numbers. It is a simplified version of Problem 1. However, while the resolution of the latter depends on combinations of univariate separation bounds, Problem 2 depends on successive applications of aggregate multivariate separation bounds and applications of Thm. 3. For this and for making the presentation easier for the reader we present both approaches.

We also estimate the expected number of real roots of $B_{\alpha}$ in the case where all the polynomials $b_{i}$ have the same degree $\nu$ and their coefficients, $b_{i, j}$, are Gaussian random variables with mean zero and variance $\left(\begin{array}{l}d \\ i\end{array}\right)$. In this case the expected number of real roots is $\sqrt{d}$. We implemented our algorithms in $\mathrm{C}$ as part of the core library of MATHEMATICA for the case $B \in \mathbb{Z}[\lg (q)][x]$ where $q$ is positive rational number and we demonstrate its efficiency over various data sets. Our results support experimentally the $\sqrt{d}$ bound for the number of roots of random polynomials of this kind. Finally, we generalize our bounds to handle bivariate polynomial systems. We prove a perturbation bound for the roots of a bivariate polynomial system that is applicable to a broader context.

The rest of the paper is structured as follows. First we introduce our notation and in Section 2 we present the main tools that we will use throughout the paper. In Section 3 we present an algorithm for tackling Problem 1 as well as its complexity analysis, experimental results and the bound for the expected number of real roots. We present a more general version of Problem 1 in Section 5 and the extension to bivariate polynomial systems in Section 6 .

Notation. In what follows $\mathcal{O}_{B}$, resp. $\mathcal{O}$, means bit, resp. arithmetic, complexity and the $\widetilde{\mathcal{O}}_{B}$, resp. $\widetilde{\mathcal{O}}$, notation means that we are ignoring logarithmic factors, see [38, Definition 25.8]. For a polynomial $A=\sum_{i=0}^{d} a_{i} x^{i} \in \mathbb{Z}[x], \operatorname{deg}(A)=d$ denotes its degree and $\mathcal{L}(A)=\tau$ the maximum bitsize of its coefficients, including a bit for the sign. For $a \in \mathbb{Q}, \mathcal{L}(a) \geq 1$ is the maximum bitsize of the numerator and the denominator. We write $\Delta_{\alpha}(A)$ to denote the minimum distance between a root $\alpha$ of a polynomial $A$ and any other root; we also use $\Delta(\alpha)$ is $A$ is clear form the context; We also use $\Delta_{i}$ instead of $\Delta\left(\alpha_{i}\right)$, where $\alpha_{i}$ is a root of $A$ and $1 \leq i \leq \operatorname{deg}(A) . \Delta(A)=\min _{\alpha} \Delta_{\alpha}(A)$ is the separation bound, that is the minimum distance between all the roots of $A$, and $\Sigma(A)=-\sum_{i=1}^{n} \lg \Delta_{i}(A)$. The Mahler measure of $A$ is 
$\mathcal{M}(A)=a_{d} \prod_{|\alpha| \geq 1}|\alpha|$, where $\alpha$ runs through the complex roots of $A$. If $A \in \mathbb{Z}[x]$ and $\mathcal{L}(A)=\tau$, then $\mathcal{M}(A) \leq\|A\|_{2} \leq \sqrt{d+1}\|A\|_{\infty}=2^{\tau} \sqrt{d+1}$ [28, page 152]. We denote by $\lg (\cdot)$, resp. $\ln (\cdot)$, the logarithm with base 2 , resp. $e$. Let $L_{\alpha}=\lg (\alpha)$, where $\alpha$ is a positive algebraic number, and $L_{H}=\lg A\left(\gamma_{1}, \gamma_{2}\right)$, where $\gamma_{\{1,2\}}$ are real algebraic and $A$ is a bivariate homogeneous polynomial and $A\left(\gamma_{1}, \gamma_{2}\right)>0$.

\section{Preliminaries}

Real algebraic numbers are the real roots of univariate polynomials with integer coefficients; we denote their set by $\mathbb{R}_{\mathrm{alg}}$. We represent them using the isolating interval representation. If $\alpha \in \mathbb{R}_{\mathrm{alg}}$ then the representation consists of a square-free polynomial with integer coefficients, $A \in \mathbb{Z}[x]$, that has $\alpha$ as a real root, and an isolating interval with rational endpoints, $\mathcal{J}=\left[\mathrm{a}_{1}, \mathrm{a}_{2}\right]$, that contains $\alpha$ and no other root of the polynomial. We write $\alpha \cong(A, \mathcal{J})$. Such a representation could be also used to represent the real roots of polynomials with real numbers as coefficients, provided that there is an algorithm for isolating them.

The following proposition provides upper and aggregate bounds for the roots of a univariate polynomial. Various versions of the proposition could be found, e.g. [13, 10, 37]. The aggregate version of Eq. (2) comes from a simplified version of [19, Thm. 11].

Proposition 1 (DMM $)$. Let $f=\sum_{i=0}^{d} a_{i} x^{i} \in \mathbb{R}[x]$ be a univariate polynomial of degree $d$ such that $a_{d} a_{0} \neq 0$. The distinct roots of $f$ are $\alpha_{1}, \ldots, \alpha_{r}$. For any root $\alpha_{k}$ it holds

$$
\frac{\left|a_{0}\right|}{2\|f\|_{\infty}} \leq\left|\alpha_{k}\right| \leq 2 \frac{\|f\|_{\infty}}{\left|a_{d}\right|}
$$

Let $K$ be any subset of $\{1, \ldots, r\}$ with cardinality $|K|$. Then

$$
\prod_{k \in K} \Delta_{k} \geq 2^{-4 d \lg d} \mathcal{M}(f)^{-2(r-1)}\left|\mathbf{s r}_{r}\left(f, f^{\prime}\right)\right|
$$

where $\mathbf{s r}_{r}\left(f, f^{\prime}\right)$ is the $r$-th subresultant coefficient of the subresultant sequence of $f$ and its derivative $f^{\prime}$.

The following lemma provides a lower bound on the evaluation of a polynomial that depends on the closest root and on the aggregate separation bound of the polynomial. For another proof with slightly different bounds, suggested by one of the reviewers, we refer the reader to the appendix.

Lemma 2. Let $L \in \mathbb{C}$ and $\gamma_{1}$ the root of the square-free polynomial $f$ that is closest to $L$. Then

$$
|f(L)| \geq\left|a_{d}\right|^{7}\left|L-\gamma_{1}\right|^{6} \mathcal{M}(f)^{-6} 2^{\lg \prod_{i} \Delta_{i}-6} .
$$

Proof: There are at most six roots of $f$ such that $\left|L-\gamma_{i}\right| \leq\left|\gamma_{i}-\gamma_{c_{i}}\right|=\Delta_{i}$, where $\gamma_{c_{i}}$ is the root closest to $\gamma_{i}$. This is a consequence of the vertex degree of planar nearest neighbor graphs [18]. Wlog let them be the first six ones. Then

$$
\begin{aligned}
|f(L)| & =\left|a_{d}\right| \prod_{i=1}^{d}\left|L-\gamma_{i}\right|=\left|a_{d}\right| \prod_{i=1}^{6}\left|L-\gamma_{i}\right| \prod_{j=7}^{d}\left|L-\gamma_{j}\right| \\
& \geq\left|a_{d}\right|\left|L-\gamma_{1}\right|^{6} \frac{1}{\prod_{i=1}^{6} \Delta_{i}} \prod_{j=1}^{d} \Delta_{j} \\
& \geq\left|a_{d}\right|^{7}\left|L-\gamma_{1}\right|^{6} \mathcal{M}(f)^{-6} 2^{\lg \prod_{i} \Delta_{i}-6} .
\end{aligned}
$$

For the last inequality we use $\Delta_{i} \leq 2 \mathcal{M}(f) /\left|a_{d}\right|$, that in turn relies on $\Delta_{i}=\left|\gamma_{i}-\gamma_{c_{i}}\right| \leq\left|\gamma_{i}\right|+\left|\gamma_{c_{i}}\right| \leq$ $2 \mathcal{M}(f) /\left|a_{d}\right|$. 
In the sequel we will use the previous lemma in conjunction with Thm. 3 and almost always $L$ will be the logarithm of an algebraic number. It might be the case that $L$ is a root of $f$ and thus the evaluation $f(L)$ is zero. However, we omit this case as it can be detected rather easily and does not affect in any case the complexity of the algorithms that we consider.

We will also need the following theorem, due to Mignotte and Waldschmidt [29]. It provides an effective lower bound on a homogeneous linear form with two logarithms of (real) algebraic numbers with algebraic coefficients. This result generalizes a result by Gel'fond. A generalization that handles general linear forms is due to Baker, e.g. [3]. In what follows by the height of an algebraic number, $\alpha$, we mean the height of the minimum polynomial of $\alpha$.

Theorem 3. [29] Let $\Lambda=\beta \log \left(\alpha_{1}\right)-\log \left(\alpha_{2}\right)$, where $\log$ is any determination of the logarithm, and $\beta, \alpha_{1}, \alpha_{2}$ are three non-zero algebraic numbers of degrees $D_{0}, D_{1}, D_{2}$, respectively. Let $A_{i}$ be a bound on the height of $\alpha_{i}$ such that $\exp \left(\left|\log \left(\alpha_{i}\right)\right|\right) \leq A_{i}$, for $i \in\{1,2\}$. B is an upper bound on the height of $\beta$ and $e^{D_{0}}$. If $D$ is the degree over $\mathbb{Q}$ of the field $\mathbb{Q}\left(\beta, \alpha_{1}, \alpha_{2}\right)$, and $T=\ln (B)+\ln \ln \left(A_{1}\right)+\ln \ln \left(A_{2}\right)+\ln (D)$, then if $\Lambda \neq 0$, then $|\Lambda|>\exp \left(-5 \cdot 10^{10} \cdot D^{4} \cdot \ln \left(A_{1}\right) \cdot \ln \left(A_{2}\right) \cdot T^{2}\right)$.

\section{An algorithm for $B_{\alpha}$}

In what follows we assume that $L_{\alpha}$ is indeed a transcendental number. This could be tested using Lindemann-Weierstrass theorem. The following lemma is based on arguments in [2].

Lemma 4. Let $\alpha$ be a positive real root of a univariate polynomial $A \in \mathbb{Z}[x]$ that has degree $m$ and maximum coefficient bitsize $\tau$. Then $2^{-2 \tau-m-2} \leq|\lg (\alpha)| \leq \tau+1$.

Proof: The right inequality follows from Cauchy's bound, since $|\alpha| \leq 2^{\tau+1}$.

For the left inequality, first we need to bound $|\alpha-1|$. Notice that $\alpha-1$ is a root of $\bar{A}(x)=A(x+1)$. The coefficients of $\bar{A}(x)$ are bounded by $2^{m+\tau}$. Using Cauchy's bound

$$
|\alpha-1| \geq 2^{-\tau-m-1} .
$$

Using the inequality $\left|e^{z}-1\right| \leq|z| e^{|z|}$, we get

$$
|\alpha-1| \leq\left|e^{\ln (\alpha)}-1\right| \leq \frac{|\lg (\alpha)|}{\lg (e)}|\alpha|
$$

and thus $|\lg (\alpha)| \geq 2^{-2 \tau-m-2}$, which concludes the proof.

Lemma 5. Let $b_{i}$ be as in Problem 1. If $b_{i}\left(L_{\alpha}\right) \neq 0$, then

$$
2^{-\widetilde{\mathcal{O}}\left(m^{4} \nu^{4} \tau\left(\tau^{2}+\nu^{2}\right)\right)} \leq\left|b_{i}\left(L_{\alpha}\right)\right| \leq 2^{\widetilde{\mathcal{O}}(\nu+\tau)} .
$$

Proof: To bound $b_{i}$ we proceed as follows:

$$
\begin{aligned}
\left|b_{i}\left(L_{\alpha}\right)\right| & =\left|\sum_{j=0}^{\nu} b_{i, j} L_{\alpha}^{j}\right| \leq 2^{\tau} \sum_{j=0}^{\nu}\left|L_{\alpha}\right|^{j} \leq 2^{\tau} \sum_{j=0}^{\nu}(\tau+1)^{j} \\
& \leq 2^{\tau+\nu+1} \tau^{\nu+1} \leq 2^{\tau+2 \nu \lg (2 \tau)} .
\end{aligned}
$$

To compute a lower bound for $\left|b_{i}\left(L_{\alpha}\right)\right|$ we assume that $\beta_{i, 1}$ is the root of $b_{i}(y)$ closest to $L_{\alpha}$ and we apply Lemma 2, i.e.

$$
\left|b_{i}\left(L_{\alpha}\right)\right|>\left|b_{i, \nu}\right|^{7}\left|L_{\alpha}-\beta_{i, 1}\right|^{6} \mathcal{M}\left(b_{i}\right)^{-6} 2^{\lg \prod_{j} \Delta_{j}\left(b_{i}\right)-6} .
$$


It holds $\left|b_{i, \nu}\right| \geq 1$; Theorem 3 implies

$$
\left|L_{\alpha}-\beta_{i, 1}\right| \geq \exp \left(c_{1} m^{4} \nu^{4} \tau(\tau+\nu+\ln (m \tau \nu))^{2}\right),
$$

where $c_{1}$ is constant that can be computed explicitly.

Landau's inequality gives $\mathcal{M}\left(b_{i}\right) \leq(\nu+1)\left\|b_{i}\right\|_{\infty} \leq 2^{\tau+\lg \nu+1}$. Finally, using Proposition 1 we have $\lg \prod_{j} \Delta_{j}\left(b_{i}\right) \geq-\mathcal{O}\left(\nu^{2}+\nu \tau+\nu \lg \nu\right)$. Combining all the inequalities we get

$$
\begin{gathered}
\left|b_{i}\left(L_{\alpha}\right)\right| \geq \exp \left(c_{2} m^{4} \nu^{4} \tau(\tau+\nu+\ln (m \tau \nu))^{2}\right), \\
\quad \text { or } \quad\left|b_{i}\left(L_{\alpha}\right)\right| \geq \exp \left(-\widetilde{\mathcal{O}}\left(m^{4} \nu^{4} \tau\left(\tau^{2}+\nu^{2}\right)\right)\right),
\end{gathered}
$$

where $c_{2}$ is constant that can be computed explicitly.

The previous lemma allows us to bound $\left\|B_{\alpha}\right\|_{2}$. Using Eq. (3) from the proof of the previous lemma we get $\left\|B_{\alpha}\right\|_{2}^{2}=\sum_{i=0}^{d}\left|b_{i}\left(L_{\alpha}\right)\right|^{2} \leq(d+1) 2^{2 \tau+2} \tau^{2 \nu+2}$, which results to

$$
\left\|B_{\alpha}\right\|_{2} \leq d 2^{\tau+1} \tau^{\nu+1}
$$

Lemma 6. Let $B_{\alpha}$ be as in Problem 1, then

$$
2^{-\widetilde{\mathcal{O}}\left(d^{6} \nu^{4} m^{4} \tau\left(\nu^{2}+\tau^{2}\right)\right)} \leq\left|\operatorname{disc}\left(B_{\alpha}\right)\right| \leq 2^{\widetilde{\mathcal{O}}\left(d \nu+d \tau+m^{4} \nu^{4} \tau\left(\tau^{2}+\nu^{2}\right)\right)} .
$$

Proof: We consider $B_{\alpha}$ as a bivariate polynomial in $\mathbb{Z}\left[L_{\alpha}, x\right]$. To bound $\left|\operatorname{disc}\left(B_{\alpha}\right)\right|$ we consider the identity

$$
\begin{aligned}
\left|\operatorname{disc}\left(B_{\alpha}\right)\right| & =\left|\frac{1}{b_{d}\left(L_{\alpha}\right)} \operatorname{res}_{x}\left(B_{\alpha}\left(L_{\alpha}, x\right), \partial B_{\alpha}\left(L_{\alpha}, x\right) / \partial x\right)\right| \\
& =\left|\frac{1}{b_{d}\left(L_{\alpha}\right)} R_{B}\left(L_{\alpha}\right)\right|
\end{aligned}
$$

where the resultant, $R_{B} \in \mathbb{Z}\left[L_{\alpha}\right]$, can be computed as the determinant of the Sylvester matrix of $B_{\alpha}\left(L_{\alpha}, x\right)$ and $\partial B_{\alpha}\left(L_{\alpha}, x\right) / \partial x$, evaluated at $L_{\alpha}$.

The Sylvester matrix is of size $(2 d-1) \times(2 d-1)$, the elements of which belong to $\mathbb{Z}\left[L_{\alpha}\right]$. The determinant consists of $(2 d-1)$ ! terms. Each term is a product of $d-1$ polynomials in $L_{\alpha}$ of degree at most $\nu$ and bitsize at most $\tau$, times a product of $d$ polynomials in $L_{\alpha}$ of degree at most $\nu-1$ and bitsize at most $\tau+\lg d$. The first product results a polynomial of degree $(d-1) \nu$ and bitsize $(d-1) \tau+(d-1) \lg d$. The second product results polynomials of degree $d(\nu-1)$ and bitsize $d \tau+d \lg (d(\nu-1))$. Thus, any term in the determinant expansion is a polynomial in $L_{\alpha}$ of degree less than $2 d \nu$ and bitsize at most $2 d \tau+6 d \lg (d \nu)$. The determinant itself is a polynomial in $L_{\alpha}$ of degree at most $2 d \nu$ and of bitsize $2 d \tau+10 d \lg (d \nu)$.

We compute an upper bound of $\left|R_{B}\left(L_{q}\right)\right|$ as follows:

$$
\left|R_{B}\left(L_{q}\right)\right| \leq 2^{2 d \tau+10 d \lg (d \nu)} \sum_{k=0}^{2 d \nu}\left|L_{\alpha}\right|^{k} \leq 2^{2 d \tau+10 d \lg (d \nu)} \tau^{2 d \nu+1} .
$$

For the lower bound, we consider $R_{B}$ as a univariate polynomial, say in $z$, and let $r$ be its leading coefficient. By $\rho_{k}$ we denote its roots. If apply Lemma 2, by assuming that $\rho_{1}$ is closest root to $L_{\alpha}$, then

$$
\left|R_{B}\left(L_{\alpha}\right)\right|>|r|^{7}\left|L_{\alpha}-\rho_{1}\right|^{6} \mathcal{M}\left(R_{B}\right)^{-6} 2^{\lg \prod_{k} \Delta_{k}\left(R_{B}\right)-6} .
$$

It holds $|r| \geq 1, \mathcal{M}\left(R_{B}\right) \leq 2^{\widetilde{\mathcal{O}}(d \tau)}$, and $-\lg \prod_{k} \Delta_{k}\left(R_{B}\right)=\mathcal{O}\left(d^{2} \nu \tau+d^{2} \nu \lg (d \nu)\right)$. We also use Theorem 3

$$
\left|L_{\alpha}-\rho_{1}\right| \geq \exp \left(-\mathcal{O}\left(d^{4} \nu^{4} m^{4} \tau(d \nu+d \tau+\lg (d \nu m \tau))^{2}\right)\right) .
$$


By combining all the inequalities we get

$$
\left|R_{B}\left(L_{\alpha}\right)\right| \geq \exp \left(-\mathcal{O}\left(d^{4} \nu^{4} m^{4} \tau(d \nu+d \tau+\lg (d \nu m \tau))^{2}\right)\right)
$$

Eq. (5) with the previous inequality and Lemma 5 imply

$$
2^{-\widetilde{\mathcal{O}}\left(d^{6} \nu^{4} m^{4} \tau\left(\nu^{2}+\tau^{2}\right)\right)} \leq\left|\operatorname{disc}\left(B_{\alpha}\right)\right| \leq 2^{\widetilde{\mathcal{O}}\left(d \nu+d \tau+m^{4} \nu^{4} \tau\left(\tau^{2}+\nu^{2}\right)\right)} .
$$

which concludes the proof.

We combine Lemma 5, Lemma 6 and Eq. (4) with Proposition 1 to derive the following (separation) bounds for $B_{\alpha}$.

Lemma 7. Let $B_{\alpha}$ be as in Problem 1 and $\beta_{i}$ be its roots. Let $K$ be any subset of the roots of $B_{\alpha}$, then

$$
\begin{gathered}
2^{-\widetilde{\mathcal{O}}\left(m^{4} \nu^{4} \tau\left(\tau^{2}+\nu^{2}\right)\right)} \leq\left|\beta_{i}\right| \leq 2^{\widetilde{\mathcal{O}}\left(m^{4} \nu^{4} \tau\left(\tau^{2}+\nu^{2}\right)\right)}, \\
\Sigma\left(B_{\alpha}\right)=-\lg \prod_{i \in K} \Delta\left(\beta_{i}\right)=\widetilde{\mathcal{O}}\left(d^{6} \nu^{4} m^{4} \tau\left(\nu^{2}+\tau^{2}\right)\right) .
\end{gathered}
$$

\subsection{Isolating the real roots of $B_{\alpha}$}

The main idea behind the algorithm for isolating the real roots of $B_{\alpha}$ is to approximate its coefficients up to a specified accuracy so that the resulting approximate polynomial, $\widetilde{B}_{\alpha}$, has real roots that are close to the real roots of $B_{\alpha}$. We isolate the real roots of $\widetilde{B}_{\alpha}$ and the approximation is such that it guarantees that the resulting isolating intervals are also isolating intervals for the real roots of $B_{\alpha}$. Several approaches are known in this context [33, 34, 30, 26], We follow [27, Theorem 3].

We divide by the leading coefficient to make the polynomial monic. As stated in Problem 1, the polynomials $b_{i} \in \mathbb{Z}[y]$ have coefficients of maximum bitsize bounded by $\tau$ and degree bounded by $\nu$.

Let $\sigma$ be such that $\left|\frac{b_{i}\left(L_{\alpha}\right)}{b_{d}\left(L_{\alpha}\right)}\right| \leq 2^{\sigma}$ and $\rho$ such that $\rho=\max _{j}\left\{1, \max \left\{1,|\log | \beta_{j}||\right\}\right\}$, that is a $\operatorname{logarithmic}$ root bound for the roots of $B_{\alpha}$.

If we approximate the coefficients of $B_{\alpha}$ up to accuracy $\mathcal{O}\left(d \rho+\Sigma\left(B_{\alpha}\right)\right)$, then we can approximate the roots (of $\widetilde{B}_{\alpha}$ ) in $\widetilde{\mathcal{O}}_{B}\left(d^{3}+d^{2} \sigma+d \Sigma\left(B_{\alpha}\right)\right.$ ). In this way the number of real roots of $\widetilde{B}_{\alpha}$ is the same as the number of real roots of $B_{\alpha}$. Moreover, from the isolating intervals of $\widetilde{B}_{\alpha}$ we can derive isolating intervals for the roots of $B_{\alpha}$. We refer the reader to [27] for a comprehensive treatment.

We bound the various quantities. Lemma 7 indicates that

$$
\Sigma\left(B_{\alpha}\right)=\widetilde{\mathcal{O}}\left(d^{6} \nu^{4} m^{4} \tau\left(\nu^{2}+\tau^{2}\right)\right)
$$

To bound $\sigma$ we use Lemma 5 and so, for all $i,\left|\frac{b_{i}\left(L_{\alpha}\right)}{b_{d}\left(L_{\alpha}\right)}\right| \leq 2^{\widetilde{\mathcal{O}}\left(m^{4} \nu^{4} \tau\left(\tau^{2}+\nu^{2}\right)\right)}$. And thus

$$
\sigma=\widetilde{\mathcal{O}}\left(m^{4} \nu^{4} \tau\left(\tau^{2}+\nu^{2}\right)\right)
$$

The same bound holds for $\rho$. Hence we need to approximate the coefficients of $B_{\alpha}$ up to accuracy

$$
\widetilde{\mathcal{O}}\left(d \rho+\Sigma\left(B_{\alpha}\right)\right)=\widetilde{\mathcal{O}}\left(d^{6} \nu^{4} m^{4} \tau\left(\nu^{2}+\tau^{2}\right)\right) .
$$

We can isolate the real roots in $\widetilde{\mathcal{O}}\left(d^{3}+d^{7} \nu^{4} m^{4} \tau\left(\nu^{2}+\tau^{2}\right)\right)$.

It remains to estimate the cost of obtaining the approximation on the coefficients of $B_{\alpha}$, that is successive approximations of $b_{i}\left(L_{\alpha}\right) / b_{d}\left(L_{\alpha}\right)$ up to accuracy of $\mathcal{O}\left(d \rho+\Sigma\left(B_{\alpha}\right)\right)$ bits after the binary point. Since $\left|b_{i}\left(L_{\alpha}\right) / b_{d}\left(L_{\alpha}\right)\right| \leq 2^{\sigma}$, to approximate each fraction, for $0 \leq i \leq d-1$, to desired accuracy $\ell$, it is sufficient to approximate $b_{i}\left(L_{\alpha}\right)$, for $0 \leq i \leq d$, up to precision $\mathcal{O}(\ell+\sigma)$. 
The algorithm requires approximation of $b_{i}\left(L_{\alpha}\right)$, for $0 \leq i \leq d$, to precision $\mathcal{O}\left(d \rho+\Sigma\left(B_{\alpha}\right)+\sigma\right)$. Hence, it is sufficient to approximate $b_{i}\left(L_{q}\right)$ to accuracy $\widetilde{\mathcal{O}}\left(d^{6} \nu^{4} m^{4} \tau\left(\nu^{2}+\tau^{2}\right)\right)$.

Approximation of $L_{\alpha}$ to accuracy of $t>0$ bits yields an approximation of $b_{i, j} L_{\alpha}^{j}$ to accuracy of at least

$$
t-\lg \left|b_{i, j}\right|-\lg (j)-(j-1) \lg \left|2 L_{\alpha}\right| \geq t-\tau-\lg (\nu)-\nu(\lg (\tau)+1)
$$

bits and an approximation of $b_{i}\left(L_{\alpha}\right)$ to accuracy of at least $t-\tau-2 \lg (\nu)-\nu(\lg (\tau)+1)$ bits. Therefore, we need an approximation of $\lg (\alpha)$ up to $t=\widetilde{\mathcal{O}}\left(d^{6} \nu^{4} m^{4} \tau\left(\nu^{2}+\tau^{2}\right)\right)$ bits.

For this we need to approximate $\alpha$ up to this accuracy and then evaluate $\lg (\alpha)$. The cost of the first operation is $\widetilde{\mathcal{O}}_{B}\left(m^{2} \tau+m t\right)$ [31]. The cost of approximating the logarithm up to $t$ bits is quasi-linear $\widetilde{\mathcal{O}}_{B}(t)$ [7], see also [8] and references therein.

After we have obtained the approximation of $L_{\alpha}$, say $\tilde{L}$ we need construct the approximated coefficients of $B_{\alpha}$ by evaluating the polynomials $b_{i}$ (of degree $\nu$ ) at $\tilde{L}$; there are $d+1$ polynomials. Each evaluation costs $\widetilde{\mathcal{O}}_{B}(\nu t)[5]$ and so the overall cost is $\widetilde{\mathcal{O}}_{B}(d \nu t)=\widetilde{\mathcal{O}}_{B}\left(d^{7} \nu^{5} m^{4} \tau\left(\nu^{2}+\tau^{2}\right)\right)$.

Theorem 8. The Boolean complexity of isolating the real roots of $B_{\alpha}$ of Problem 1 is $\widetilde{\mathcal{O}}\left(d^{7} \nu^{5} m^{4} \tau\left(\nu^{2}+\tau^{2}\right)\right)$.

If we want to drop the assumption that the polynomial $B_{\alpha}$ is square-free, then we can apply a subresultant-based algorithm to compute its square-free part, or its square-free factorization [38, ]. To apply such algorithms we need to check if the leading coefficient of the polynomial in the subresultant sequence is zero or not. These coefficients are polynomials in $\mathbb{Z}\left[L_{\alpha}\right]$. Therefore, the basic operation needed is to compute the sign of a univariate polynomial evaluated at the logarithm of an algebraic number. To accomplish such an operation we need the bounds of Lem. 5. The exact complexity of the complete algorithm for square-free factorization of polynomials in $\mathbb{Z}\left[L_{\alpha}\right][x]$ is beyond the scope of this paper.

\section{Experiments}

We present experimental results for an implementation of the algorithm isolating roots of the polynomial in Problem 1 in the special case where the algebraic number $\alpha$ is a rational. The algorithm has been implemented in $\mathrm{C}$ as a part of the Mathematica system. We have implemented the modified version of Descartes' algorithm due to Sagraloff [33], that applies to polynomials with bitstream coefficients, see also $[15,26]$, and we adapted our bounds to it. We assume that the input polynomial is square-free. If it is not the algorithm does not terminate, or we can hard-code an upper bound for the number of iterations. The theoretical complexity of the algorithm is worse by factor than the complexity the algorithm that we presented in the previous section, but its implementation is much easier.

The experiments have been run on a 64-bit Windows virtual machine with a $3 \mathrm{GHz}$ Intel Core i7 processor and $6 \mathrm{~GB}$ of RAM. The timings are in given seconds. The MATHEMATICA code that we used to perform the experiments is publicly available ${ }^{1}$.

Example 9. (Random polynomials with uniformly distributed coefficients) For given values of $d, \nu$ and $\tau$ each instance (polynomial) was generated by selecting integer coefficients $b_{i, j}$ randomly w.r.t. the uniform distribution in $\mathbb{Z} \cap\left[-2^{\tau-1}, 2^{\tau-1}\right]$ and a positive rational number $\alpha \neq 1$ with $\mathcal{L}(\alpha) \leq \tau$. Each timing is an average for 10 randomly generated problems. The results are in Table 1 and 2 .

Applying a least-squares fit to the experimental data yields proportionality of the computation time to $d^{1.4} \nu^{0.8}$. There is very little dependence of the computation time on the value of $\tau$ (see also the next section).

\footnotetext{
${ }^{1}$ http://members.wolfram.com/adams/LogRootIsolExamples.txt
} 


\begin{tabular}{|c||c|c|c|c|c|c|c|}
\hline$d \backslash \nu$ & 10 & 20 & 50 & 100 & 200 & 500 & 1000 \\
\hline \hline 10 & 0.006 & 0.011 & 0.027 & 0.060 & 0.122 & 0.358 & 0.857 \\
\hline 20 & 0.015 & 0.025 & 0.058 & 0.110 & 0.235 & 0.678 & 1.53 \\
\hline 50 & 0.042 & 0.068 & 0.142 & 0.272 & 0.581 & 1.61 & 3.56 \\
\hline 100 & 0.116 & 0.164 & 0.339 & 0.640 & 1.19 & 3.14 & 7.65 \\
\hline 200 & 0.496 & 0.516 & 0.900 & 1.65 & 2.76 & 6.41 & 16.7 \\
\hline 500 & 3.43 & 4.53 & 5.30 & 6.52 & 10.4 & 21.5 & 54.6 \\
\hline 1000 & 25.5 & 23.1 & 27.7 & 36.8 & 45.7 & 79.9 & 173 \\
\hline
\end{tabular}

Table 1. Uniformly distributed coefficients, $\tau=10$

\begin{tabular}{|c||c|c|c|c|c|c|c|}
\hline$d \backslash \nu$ & 10 & 20 & 50 & 100 & 200 & 500 & 1000 \\
\hline \hline 10 & 0.006 & 0.011 & 0.028 & 0.054 & 0.120 & 0.362 & 0.883 \\
\hline 20 & 0.015 & 0.026 & 0.060 & 0.116 & 0.237 & 0.809 & 1.65 \\
\hline 50 & 0.045 & 0.072 & 0.157 & 0.299 & 0.671 & 1.74 & 3.98 \\
\hline 100 & 0.136 & 0.200 & 0.356 & 0.759 & 1.37 & 3.41 & 7.78 \\
\hline 200 & 0.442 & 0.605 & 0.985 & 1.62 & 2.84 & 7.25 & 17.9 \\
\hline 500 & 4.30 & 4.48 & 5.95 & 7.55 & 12.6 & 25.4 & 60.1 \\
\hline 1000 & 20.5 & 30.4 & 30.4 & 34.8 & 44.7 & 81.4 & 183 \\
\hline
\end{tabular}

Table 2. Uniformly distributed coefficients, $\tau=1000$

Example 10. (Random polynomials with Gaussian distribution of coefficients) For given values of $d$ and $\nu$ each problem was generated by setting $\alpha=3$ and selecting coefficients $b_{i, j}$ as nearest integers to real numbers selected randomly w.r.t. the Gaussian distribution with mean 0 and variance $\left(\begin{array}{l}d \\ i\end{array}\right)$. Each result is an average for 100 randomly generated problems. For each value $d$ and $\nu$ the upper section gives the computation time and the lower section gives the number of real roots. The results are in Table 3.

Applying a least-squares fit to the experimental data yields proportionality of the computation time to $d^{1.7} \nu^{0.8}$. The average number of roots is, as expected, close to $\sqrt{d}$.

\subsection{Random polynomials}

We were not able to construct polynomials that achieve the separation bounds of Lemma 7 . It is not clear whether the effective lower bounds of Theorem 3 are tight. Our experimental results of the previous section suggest that this is not the case for random polynomials. In addition, this observation triggers the question of estimating the average behavior of the separation bounds. The first step is to estimate the expected number of real roots of $B_{q}$, when its coefficients are random variables.

Proposition 11. [14] Let $v(t)=\left(f_{0}(t), \ldots, f_{n}(t)\right)^{\top}$ be a vector of differentiable functions and $c_{0}, \ldots, c_{n}$ elements of a multivariate normal distribution with zero mean and covariance matrix $C$. The expected number of real zeros on an interval (or a measurable set) $I$ of the equation $c_{0} f_{0}(t)+\cdots+c_{n} f_{n}(t)=0$, for $w(t)=C^{1 / 2} v(t)$, is

$$
\int_{I} \frac{1}{\pi}\left\|\boldsymbol{w}^{\prime}(t)\right\| d t, \boldsymbol{w}=w(t) /\|w(t)\|
$$

In logarithmic derivative notation it is

$$
\frac{1}{\pi} \int_{I} \sqrt{\left.\frac{\partial^{2}}{\partial x \partial y} \log \left(v(x)^{\top} C v(y)\right)\right|_{x=y=t}} d t .
$$




\begin{tabular}{|c||c|c|c|c|c|c|c|}
\hline$d \backslash \nu$ & 10 & 20 & 50 & 100 & 200 & 500 & 1000 \\
\hline \hline \multirow{2}{*}{10} & 0.004 & 0.005 & 0.013 & 0.028 & 0.072 & 0.290 & 0.992 \\
\cline { 2 - 8 } & 3.20 & 3.06 & 3.28 & 3.14 & 3.30 & 3.10 & 3.22 \\
\hline \multirow{2}{*}{20} & 0.013 & 0.022 & 0.050 & 0.109 & 0.239 & 0.902 & 2.07 \\
\cline { 2 - 8 } & 4.40 & 4.18 & 4.56 & 4.48 & 4.66 & 4.28 & 4.14 \\
\hline \multirow{2}{*}{50} & 0.080 & 0.118 & 0.191 & 0.406 & 0.794 & 2.34 & 5.33 \\
\cline { 2 - 8 } & 7.46 & 7.22 & 6.74 & 6.96 & 7.12 & 7.06 & 6.86 \\
\hline \multirow{2}{*}{100} & 0.309 & 0.384 & 0.596 & 0.477 & 1.06 & 2.03 & 5.07 \\
\cline { 2 - 8 } & 9.92 & 10.12 & 9.98 & 10.12 & 9.90 & 10.44 & 10.02 \\
\hline \multirow{2}{*}{200} & 1.75 & 2.19 & 2.49 & 4.10 & 6.56 & 9.42 & 18.8 \\
\cline { 2 - 8 } & 13.98 & 14.02 & 13.78 & 14.36 & 13.98 & 14.24 & 13.92 \\
\hline \multirow{2}{*}{500} & 32.4 & 32.9 & 34.4 & 35.9 & 39.9 & 51.7 & 88.5 \\
\cline { 2 - 8 } & 22.92 & 22.50 & 22.46 & 22.10 & 21.92 & 22.72 & 22.80 \\
\hline
\end{tabular}

Table 3. Gaussian distribution of coefficients

We fix a $\operatorname{logarithm} L$. For example $L=\lg (q)$ for a (fixed) positive rational number $q$, different from 0 and 1 , or $L=\lg (\alpha)$, where $\alpha$ is a positive real algebraic number. Consider the polynomials $b_{i}=\sum_{j=0}^{\nu} b_{i, j} L^{j}$ where each of $b_{i, j}$ is a Gaussian random variable with mean zero and variance $\left(\begin{array}{l}d \\ i\end{array}\right)$. We denote this by $b_{i, j} \sim N\left(0,\left(\begin{array}{c}d \\ i\end{array}\right)\right)$. Then

$$
b_{i} \sim N\left(0,\left(\begin{array}{l}
d \\
i
\end{array}\right) \sum_{j=0}^{\nu} L^{2 j}\right)=N\left(0,\left(\begin{array}{l}
d \\
i
\end{array}\right) \ell\right) .
$$

In our case $v(x)^{\top} C v(y)=\ell(1+x y)^{d}$, and the integral of Proposition 11 yields

$$
\frac{1}{\pi} \int_{-\infty}^{\infty} \sqrt{\left.\frac{\partial^{2}}{\partial x \partial y} \log \ell(1+x y)^{d}\right|_{x=y=t}} d t=\sqrt{d} .
$$

This leads to the following lemma:

Lemma 12. Let $B_{\alpha}$ as in Prob. 1 with a fixed $\alpha$. Let all $b_{i}$ have the same degree $\nu$ and $b_{i, j} \sim N\left(0,\left(\begin{array}{c}d \\ i\end{array}\right)\right)$. Then the expected number of real roots of $B_{q}$ is $\sqrt{d}$.

Following, mutatis mutandis, the analysis of [16, Lemma 3.2] the previous lemma allows us to compute the distribution of the real roots and eventually to estimate the expected separation bound; which is $\mathrm{E}\left[-\lg \Delta\left(B_{\alpha}\right)\right]=\mathcal{O}(\lg d)$ (for the aforementioned distribution of the coefficients), for the real roots. This is far from the worst case proved in Lemma 7 but agrees with the running times of our implementation in Section 4. The bigger the (actual) separation bound, the less bits we need to isolate the real roots, and so the faster the algorithms perform. For estimating the expected separation bounds for the complex roots, we need to compute (expected) lower bounds on the discriminant. We are not aware of such bounds.

\section{$5 \quad$ A generalization}

We present a generalization of Problem 1 where the argument of the logarithm is a homogeneous bivariate polynomial evaluated at two real algebraic numbers. As in the case of Problem 1 we rely on Thm. 3 for computing the various upper and lower bounds.

The precise problem definition is as follows:

Problem 2. Consider the square-free $B_{H}=\sum_{i=0}^{d} b_{i} x^{i}$, where $b_{i}=\sum_{j=0}^{\nu} b_{i, j}\left(\lg \left(A\left(\gamma_{1}, \gamma_{2}\right)\right)\right)^{j}, b_{i, j} \in \mathbb{Z}$, $\mathcal{L}\left(b_{i, j}\right) \leq \tau, A \in \mathbb{Z}\left[y_{1}, y_{2}\right]$ is a homogeneous polynomial of degree $m$ and $\mathcal{L}(A)=\tau$ and $\gamma_{1}$, resp. $\gamma_{2}$, is a real 
root of a polynomial $C_{1} \in \mathbb{Z}[y]$, resp. $C_{2} \in \mathbb{Z}[y]$, of degree $n$ and $\mathcal{L}\left(C_{\{1,2\}}\right)=\tau$. We assume $A\left(\gamma_{1}, \gamma\right)>0$ and $A\left(\gamma_{1}, \gamma_{2}\right) \neq 1$. What is the Boolean complexity of isolating the real roots of $B_{H}$ ?

We should warn the reader that the constants in the various bounds in the sequel are not the best possible.

Lemma 13. Let $A \in \mathbb{Z}\left[y_{1}, y_{2}\right]$ be a homogeneous polynomial of degree $m$ and $\mathcal{L}(A)=\tau$ and $\gamma_{1}$, resp. $\gamma_{2}$, be the positive real root of a polynomial $C_{1} \in \mathbb{Z}[y]$, resp. $C_{2} \in \mathbb{Z}[y]$, that is of degree $n$ and $\mathcal{L}(C)=\tau$. Then $2^{-3 n^{2} \tau-5 n^{2} \lg (m n)-4 m \tau} \leq\left|\lg A\left(\gamma_{1}, \gamma_{2}\right)\right| \leq 4 m \tau$.

Proof: Assume for the moment that we know positive integers $t$ and $T$ such that $\left|A\left(\gamma_{1}, \gamma_{2}\right)\right| \leq 2^{T}$ and $\left|A\left(\gamma_{1}, \gamma_{2}\right)-1\right| \geq 2^{-t}$. Then from the inequality $\left|e^{z}-1\right| \leq|z| e^{|z|}$ we deduce

$$
\begin{gathered}
\left|A\left(\gamma_{1}, \gamma_{2}\right)-1\right| \leq\left|\ln A\left(\gamma_{1}, \gamma_{2}\right)\right| e^{\left|\ln A\left(\gamma_{1}, \gamma_{2}\right)\right|} \Rightarrow \\
\left|A\left(\gamma_{1}, \gamma_{2}\right)-1\right| \leq \frac{\left|\lg A\left(\gamma_{1}, \gamma_{2}\right)\right|}{\lg (e)}\left|A\left(\gamma_{1}, \gamma_{2}\right)\right| \Rightarrow \\
2^{-t-1} \leq\left|A\left(\gamma_{1}, \gamma_{2}\right)-1\right| \leq\left|\lg A\left(\gamma_{1}, \gamma_{2}\right)\right| 2^{T} \Rightarrow \\
2^{-t-T-1} \leq\left|\lg A\left(\gamma_{1}, \gamma_{2}\right)\right| .
\end{gathered}
$$

It remains to specify $t$ and $T$. For the real algebraic numbers $\gamma_{1}$ and $\gamma_{2}$ it holds

$$
2^{-\tau-1} \leq\left|\gamma_{\{1,2\}}\right| \leq 2^{\tau+1}
$$

We bound $T$ as follows:

$$
\left|A\left(\gamma_{1}, \gamma_{2}\right)\right| \leq\left|\sum_{i=0}^{m} a_{i} \gamma_{1}^{i} \gamma_{2}^{m-i}\right| \leq \sum_{i=0}^{m} 2^{\tau} 2^{m \tau}
$$

and so

$$
\left|\lg A\left(\gamma_{1}, \gamma_{2}\right)\right| \leq(m+1) \tau+\lg (m+1)=T .
$$

We choose $T=4 m \tau=\mathcal{O}(m \tau)$ to simplify the calculations.

To compute a bound for $t$ we consider the polynomial $\bar{A}\left(y_{1}, y_{2}\right)=A\left(y_{1}, y_{2}\right)-1$ and the following polynomial system:

$$
\begin{cases}F_{1}=z-\left[A\left(y_{1}, y_{2}\right)-1\right] & =0 \\ F_{2}=C_{1}\left(y_{1}\right) & =0 \\ F_{3}=C_{2}\left(y_{2}\right) & =0\end{cases}
$$

We will use a similar system in the sequel so we present various quantities that are related to it. For further details on DMM we refer the reader to [17].

A lower bound on $z$ provides us with a lower bound for $t$. To compute a bound for $z$ we use the DMM bound from [17, Thm.3].

Let $\mathcal{D}$ be the mixed volume of the system, $\mathrm{MV}_{i}$ the mixed volume of the system if we discard the $i$-th polynomial, \# $\left(Q_{i}\right)$ the number of integer points of the Newton polytope of the $i$-th polynomial, for $1 \leq i \leq 3, \varrho=\prod_{i=1}^{3}\left(\# Q_{i}\right)^{\mathrm{MV}_{i}}$, and $\mathcal{C}=\prod_{i=1}^{3}\left\|F_{i}\right\|_{\infty}^{\mathrm{MV}_{i}}$.

The univariate polynomial that has the $z$-coordinates of the solution set of the system as roots, we call them $\zeta$, has degree $\mathcal{D}$ and maximum coefficient bitsize $\varrho 2^{\mathcal{D}} \mathcal{C}$. It holds $|\zeta| \geq\left(\varrho 2^{\mathcal{D}} \mathcal{C}\right)^{-1}$. In our case

$$
\begin{array}{r}
\mathcal{D}=n^{2}, \mathrm{MV}_{1}=n^{2}, \mathrm{MV}_{2}=\mathrm{MV}_{3}=n \\
\left(\# Q_{1}\right)=m+1,\left(\# Q_{2}\right)=\left(\# Q_{3}\right)=n+1, \\
\varrho=(m+1)^{n^{2}}(n+1)^{2 n}, \mathcal{C} \leq 2^{\tau\left(n^{2}+2 n\right)}
\end{array}
$$

Notice that it is exactly the use of mixed volume that allows us to take $\mathcal{D}=n^{2}$ instead of $m n^{2}$ which is the Bézout bound. 
The lower bound for $\zeta$ becomes

$$
|\zeta| \geq 2^{-\left(n^{2}+n^{2} \lg (m+1)+2 n \lg (n+1)+\tau\left(n^{2}+2 n\right)\right)},
$$

and hence

$$
t=n^{2}+n^{2} \lg (m+1)+2 n \lg (n+1)+\tau\left(n^{2}+2 n\right) .
$$

We choose $t=3 n^{2} \tau+5 n^{2} \lg (m n)=\widetilde{\mathcal{O}}\left(n^{2} \tau\right)$.

Lemma 14. Let $b_{i}$ be as in Problem 2. If $b_{i}\left(L_{H}\right) \neq 0$, then $2^{-\widetilde{\mathcal{O}}\left(n^{10} \nu^{4} \tau\left(\tau^{2}+\nu^{2}\right)\right)} \leq\left|b_{i}\left(L_{H}\right)\right| \leq 2^{\widetilde{\mathcal{O}}(\nu+\tau)}$.

Proof: For all $i$ it holds

$$
\left|b_{i}\left(L_{H}\right)\right|=\left|\sum_{j=0}^{\nu} b_{i, j} L_{H}^{j}\right| \leq \sum_{j=0}^{\nu} 2^{\tau}(4 m \tau)^{j} \leq(\nu+1) 2^{\tau}(4 m \tau)^{\nu},
$$

and so

$$
\left|b_{i}\left(L_{H}\right)\right| \leq 2^{\tau+8 \nu \lg (m \tau)} .
$$

We consider $b_{i}$ as a univariate polynomial in $y$ and so $b_{i}=\sum_{j=0}^{\nu} b_{i, j} y^{i}=b_{i, \nu} \prod_{j=1}^{\nu}\left(y-\beta_{i, j}\right)$, where $\beta_{i, j}$ are its roots. Let $\beta_{i, 1}$ the root closest to $L_{H}$; we apply Lemma 2

$$
\left|b_{i}\left(L_{H}\right)\right|>\left|b_{i, \nu}\right|^{7}\left|L_{H}-\beta_{i, 1}\right|^{6} \mathcal{M}\left(b_{i}\right)^{-6} 2^{\lg \prod_{j} \Delta\left(b_{i}\right)-6} .
$$

It holds $\left|b_{i, \nu}\right| \geq 1, \mathcal{M}\left(b_{i}\right) \leq 2^{\tau+\lg \nu+1}$, and $-\lg \prod_{j} \Delta\left(b_{i}\right)=\mathcal{O}\left(\nu^{2}+\nu \tau\right)$.

To bound $\left|L_{H}-\beta_{i, 1}\right|$ we use Theorem 3. For this we need to identify the real algebraic number $A\left(\gamma_{1}, \gamma_{2}\right)$ represents. Consider the following polynomial system:

$$
\begin{cases}F_{1}=z-A\left(y_{1}, y_{2}\right) & =0 \\ F_{2}=C_{1}\left(y_{1}\right) & =0 \\ F_{3}=C_{2}\left(y_{2}\right) & =0\end{cases}
$$

The system is almost identical to the one in the proof of Lemma 13 and so we get all the (worst case) bounds from that system. If we eliminate $y_{1}$ and $y_{2}$, then we get a univariate polynomial in $z$ among the solutions of which is the real algebraic number $A\left(\gamma_{1}, \gamma_{2}\right)$. The polynomial has degree $n^{2}$ and maximum coefficient bitsize $n^{2}+n^{2} \lg (m+1)+2 n \lg (n+1)+\tau\left(n^{2}+2 n\right)=\widetilde{\mathcal{O}}\left(n^{2} \tau\right)$. Then, Thm 3 implies that

$$
\left|L_{H}-\beta_{i, j}\right| \geq \exp \left(-\mathcal{O}\left(n^{10} \nu^{4} \tau(\tau+\nu+\lg (n \nu \tau))^{2}\right) .\right.
$$

By combining all the bounds we obtain the bound $\left|b_{i}\left(L_{H}\right)\right|>2^{-\mathcal{O}\left(n^{10} \nu^{4} \tau(\tau+\nu+\lg (n \nu \tau))^{2}\right)}$, which concludes the proof.

An upper bound for $\left\|B_{H}\right\|_{2}$ is $\left\|B_{H}\right\|_{2}^{2}=\sum_{i=0}^{d}\left|b_{i}\left(L_{H}\right)\right|^{2} \Rightarrow\left\|B_{H}\right\|_{2} \leq 2^{\tau+8 \nu \lg (m \tau)+\lg (d)}$.

Lemma 15. Let $B_{H}$ be as in Problem 2, then

$$
2^{-\widetilde{\mathcal{O}}\left(d^{6} n^{8} \nu^{4} \tau\left(\nu^{2}+\tau^{2}\right)\right)} \leq\left|\operatorname{disc}\left(B_{H}\right)\right| \leq 2^{\widetilde{\mathcal{O}}\left(d \nu+d \tau+n^{10} \nu^{4} \tau\left(\tau^{2}+\nu^{2}\right)\right)} .
$$

Proof: As in the proof of Lemma 6 we consider $B_{H}$ as a bivariate polynomial in $\mathbb{Z}\left[L_{H}, x\right]$, and

$$
\begin{aligned}
\left|\operatorname{disc}\left(B_{H}\right)\right| & =\left|\frac{1}{b_{d}\left(L_{H}\right)} \operatorname{res}_{x}\left(B_{H}\left(L_{H}, x\right), \partial B_{H}\left(L_{H}, x\right) / \partial x\right)\right| \\
& =\left|\frac{1}{b_{d}\left(L_{H}\right)} R_{B}\left(L_{H}\right)\right|
\end{aligned}
$$


The resultant $R_{B} \in \mathbb{Z}\left[L_{H}\right]$ is a univariate polynomial of degree at most $2 d \nu$ and maximum coefficient bitsize $2 d \tau+10 d \lg (d \nu)$. Therefore

$$
\begin{aligned}
\left|R_{B}\left(L_{H}\right)\right| & \leq 2^{2 d \tau+10 d \lg (d \nu)} \sum_{k=0}^{2 d \nu}\left|L_{H}\right|^{k} \\
& \leq 2^{2 d \tau+10 d \lg (d \nu)}(4 m \tau)^{2 d \nu+1} .
\end{aligned}
$$

For the lower bound, let $r$ be the leading coefficient of $R_{B}$ and $\rho_{k}$ its roots. Let $\rho_{1}$ be the root closest to $L_{H}$. Then $|r| \geq 1, \mathcal{M}\left(R_{B}\right) \leq 2^{2 d \tau+12 d \lg (d \nu)},-\lg \prod_{k} \Delta\left(R_{B}\right)=\mathcal{O}\left(d^{2} \nu^{2}+d^{2} \nu \tau\right)$. The application of Theorem 3 gives us

$$
\left|L_{H}-\rho_{1}\right| \geq \exp \left(-\widetilde{\mathcal{O}}\left(d^{6} n^{8} \nu^{4} \tau\left(\nu^{2}+\tau^{2}\right)\right)\right) .
$$

Using Lemma 2 we get

$$
\left|R_{B}\left(L_{H}\right)\right|>|r|^{7}\left|L_{H}-\rho_{1}\right|^{6} \mathcal{M}\left(R_{B}\right)^{-6} 2^{\lg \prod_{k} \Delta_{k}\left(R_{B}\right)-6}
$$

and thus

$$
\left|R_{B}\left(L_{H}\right)\right| \geq \exp \left(-\widetilde{\mathcal{O}}\left(d^{6} n^{8} \nu^{4} \tau\left(\nu^{2}+\tau^{2}\right)\right)\right) .
$$

Combining Eq. (8) with the previous inequality and Lemma 14 we get

$$
2^{-\widetilde{\mathcal{O}}\left(d^{6} n^{8} \nu^{4} \tau\left(\nu^{2}+\tau^{2}\right)\right)} \leq\left|\operatorname{disc}\left(B_{H}\right)\right| \leq 2^{\widetilde{\mathcal{O}}\left(d \nu+d \tau+n^{10} \nu^{4} \tau\left(\tau^{2}+\nu^{2}\right)\right)}
$$

that concludes the proof.

Lemma 16. Let $B_{H}$ be as in Problem 2 and let $\beta_{j}$ be its roots. Then

$$
\begin{gathered}
2^{-\widetilde{\mathcal{O}}\left(n^{10} \nu^{4} \tau\left(\tau^{2}+\nu^{2}\right)\right)} \leq\left|\beta_{j}\right| \leq 2^{\widetilde{\mathcal{O}}\left(n^{10} \nu^{4} \tau\left(\tau^{2}+\nu^{2}\right)\right)} \\
\Sigma\left(B_{H}\right)=-\lg \prod_{(i, j) \in \Omega}\left|\beta_{i}-\beta_{j}\right| \widetilde{\mathcal{O}}\left(d^{6} n^{8} \nu^{4} \tau\left(\nu^{2}+\tau^{2}\right)\right) .
\end{gathered}
$$

When we have two or more logarithms and the polynomials are not homogeneous or if we have homogeneous polynomials and three or more logarithms then we are not able to compute separation bounds. In this case the separation bounds are closely connected to major open problems in number theory, like the four exponentials conjecture. For example, no effective lower bounds are known for the expression $\left|\lg \left(\alpha_{1}\right) \lg \left(\alpha_{2}\right)-\lg \left(\alpha_{3}\right) \lg \left(\alpha_{4}\right)\right|$, where $\alpha_{\{1,2,3,4\}}$ are (real) algebraic numbers.

\subsection{Isolating the real roots of $B_{H}$}

We proceed as in Section 3.1 and we use the same notation. We approximate the coefficients of $B_{H}$ up to accuracy $\mathcal{O}\left(d \rho+\Sigma\left(B_{H}\right)\right)$ and we isolate the real roots in $\widetilde{\mathcal{O}}_{B}\left(d^{3}+d^{2} \sigma+d \Sigma\left(B_{H}\right)\right)$. From Lemma 16 we get $\Sigma\left(B_{H}\right)=\widetilde{\mathcal{O}}\left(d^{6} n^{8} \nu^{4} \tau\left(\nu^{2}+\tau^{2}\right)\right)$. Moreover, $\rho=\widetilde{\mathcal{O}}\left(n^{10} \nu^{4} \tau\left(\tau^{2}+\nu^{2}\right)\right)$ and $\sigma=\widetilde{\mathcal{O}}\left(n^{10} \nu^{4} \tau\left(\tau^{2}+\nu^{2}\right)\right)$.

We need to estimate the cost of approximating $b_{i}\left(L_{H}\right) / b_{d}\left(L_{H}\right)$ up to accuracy of $\mathcal{O}\left(d \rho+\Sigma\left(B_{H}\right)\right)$ bits after the binary point. Working as in Section 3.1 we deduce that we should approximate $L_{H}=\lg A\left(\gamma_{1}, \gamma_{2}\right)$ up to precision $2^{-t}$, where $t=\mathcal{O}\left(d \rho+\Sigma\left(B_{H}\right)\right)$. The cost of this approximation is quasi-linear $\widetilde{\mathcal{O}}_{B}(t)[7]$.

In addition, we should also approximate $A\left(\gamma_{1}, \gamma_{2}\right)$ up to this accuracy. Assume that we have isolating intervals $\left[\gamma_{1}\right]$, resp. $\left[\gamma_{2}\right]$, for the real algebraic number $\gamma_{1}$, resp. $\gamma_{2}$. Let their widths be $2^{-s}$, where $s$ is a positive integer that we should determine. That is wid $\left[\gamma_{1}\right]=\operatorname{wid}\left[\gamma_{2}\right]=2^{-s}$.

Recall that $2^{-\tau} \leq\left|\gamma_{\{1,2\}}\right| \leq 2^{\tau}$ and that $A=\sum_{i=0}^{m} a_{i} y_{1}^{i} y_{2}^{m-i}$ is a homogeneous bivariate polynomial of degree $m$. 
For an expression $E$, let $[E]$ be its evaluation using interval arithmetic. Using the properties of interval arithmetic [1] we get that wid $\left[a_{i} \gamma_{1}^{i} \gamma_{2}^{m-i}\right] \leq m 2^{\tau(m-1)} 2^{-s}$, and $\operatorname{wid}\left[A\left(\gamma_{1}, \gamma_{2}\right)\right] \leq m^{2} 2^{m \tau} 2^{-s} \leq 2^{-t}$, which leads to $s=t+m \tau+2 \lg (m)=\widetilde{\mathcal{O}}\left(n^{8} \nu^{5} \tau^{3}\left(n^{2}+d^{8}\right)\right)$.

We approximate $\gamma_{1}$ and $\gamma_{2}$ up to this accuracy in $\widetilde{\mathcal{O}}_{B}\left(n^{2} \tau+n s\right)=\widetilde{\mathcal{O}}_{B}\left(n^{2} \tau+n m \tau+n t\right)[31]$.

It remains to estimate the cost of computing the approximated coefficients of $B_{H}$. After we have computed a approximation of $L_{H}$, say $\tilde{L}_{H}$, we need perform the evaluation $b_{i}\left(\tilde{L}_{H}\right)$; there are $d+1$. Each costs $\widetilde{\mathcal{O}}_{B}(\nu s)$ and the overall cost is $\widetilde{\mathcal{O}}_{B}(d \nu s)$.

Combining all the bounds we have the following theorem

Theorem 17. The Boolean complexity of isolating the real roots of $B_{H}$ of Problem 2 is $\widetilde{\mathcal{O}}_{B}\left(n^{9} \nu^{4} d^{2} \tau\left(\tau^{2}+\right.\right.$ $\left.\left.\nu^{2}\right)\left(n^{2}+d^{5}\right)+m \tau(n+d \nu)\right)$.

\section{An extension to bivariate polynomial systems}

In this section we consider bivariate polynomial systems. Let $L=L_{q}$ or $L=L_{H}$ (Section 3 and Section 5 , respectively). The problem statement is as follows:

Problem 3. Consider the, zero dimensional, polynomial system $\left(S_{L}\right) F_{1}(x, y)=F_{2}(x, y)=0$, where $F_{1,2} \in(\mathbb{Z}[L])\left[x_{1}, x_{2}\right]$ and their total degree is bounded by d. Let $L=L_{q}=\lg (q)$, resp. $L=L_{H}=\lg A\left(\gamma_{1}, \gamma_{2}\right)$, be as in Problem 1, resp. Problem 2. The coefficients of $F_{1}$ and $F_{2}$ are polynomials in $L$ of degree $\nu$ and maximum coefficient bitsize at most $\tau$. What is the Boolean complexity of isolating the real roots of $\left(S_{L}\right)$ ?

The complexity of the algorithms for solving bivariate polynomial systems depends heavily on the separation bound of the system. We present the separation bounds and we sketch the analysis of isolation process. We use the DMM bound [17]. Consider the polynomial system

$$
\left(S_{0}\right) \quad F_{1}\left(x_{1}, x_{2}\right)=F_{2}\left(x_{1}, x_{2}\right)=u-x_{1}=0
$$

where $u$ is a parameter. If we eliminate $x_{1}$ and $x_{2}$ from $\left(S_{0}\right)$ then we get a univariate polynomial in $u$, $R_{1} \in(\mathbb{Z}[L])[u]$, which is called the $u$-resultant. The DMM bound bounds the separation of $S_{L}$ using the separation bound of $R_{1}$. Asymptotically, the latter depends on a lower bound on the discriminant of its square-free part [17, Thm. 3]. Hence, it suffices to estimate this bound.

The coefficients of $R_{1}$ are of the form $\varrho_{k} c_{1}^{d} c_{2}^{d} u^{k}$, where $0 \leq k \leq d^{2}, c_{\{1,2\}}$ denotes a monomial in the coefficients of $F_{\{1,2\}}$ of total degree $d$, and $\varrho_{k}$ is an integer that depends on the integer points of the Newton polytopes of the polynomials and in our case is bounded by $\left|\varrho_{k}\right| \leq\left(d^{2}+2\right)^{2 d}$. The degree of $R_{1}$ wrt $u$ is $\mathcal{O}\left(d^{2}\right)$.

Recall that the coefficients of $F_{\{1,2\}}$ are polynomials in $L$. Thus, the coefficients of $R_{1}$ are also polynomials in $L$ of degree at most $2 d \nu$ and maximum coefficient bitsize $\widetilde{\mathcal{O}}(d \tau)$. If we compute the square-free part of $R_{1}$, then its coefficients are polynomials of degree bounded by $2 d \nu$ and of maximum coefficient bitsize bounded by $2 d \tau+10 d \lg (d)=\widetilde{\mathcal{O}}(d \tau)$ [43]. If $L=L_{\alpha}$ then we apply Lemma 6 and the logarithm of the separation bound of the system is $\widetilde{\mathcal{O}}\left(d^{7} \nu^{10} m^{4} \tau\left(\nu^{2}+d^{2} \tau^{2}\right)\right)$. We can obtain a similar bound if $L=L_{H}=\lg A\left(\gamma_{1}, \gamma_{2}\right)$ and we apply Lemma 15. In both cases, it seems that the bounds are quite pessimistic. We can also obtain the bounds by modifying accordingly the DMM bound [17].

To compute $R_{1}$ (or $R_{2}$ if we choose to eliminate $x_{2}$ ) we treat $L$ as a new variable. The projection on $x_{1}$, that is the computation of $R_{1} \operatorname{costs} \widetilde{\mathcal{O}}_{B}\left(d^{5} \nu \tau\right)$ ([12, Prop. 8 and Lemma 9]). The cost is the same for projection on the $x_{2}$-axis. Using the previous bounds and the results of Sections 3.1 and 5.1 we can isolate the roots of the two projections. For the $L_{\alpha}$ case this cost is $\widetilde{\mathcal{O}}_{B}\left(d^{2} \nu^{4}+d^{8} \nu^{12} m^{4} \tau\left(\nu^{2}+d^{2} \tau^{2}\right)\right)$. It remains to match the $x_{1}$ and $x_{2}$ coordinates. For example, we can use one of the three strategies in [12]. The main operation needed is the computation of sign of a univariate polynomial like $B_{\alpha}$ evaluated at a real algebraic number. We postpone the detailed analysis for a future communication 
Another way to solve the system is to approximate $L$ up to an accuracy, substitute this value to the polynomials $F_{\{1,2\}}$, and then solve the system. We need a perturbation bound for the roots of a bivariate system, similar to the one(s) for univariate polynomials [34, Theorem 19.1].

Theorem 18. Consider a 0 -dimensional polynomial system $F=0$, where $F=\left(F_{1}, F_{2}\right)$ and $F_{\{1,2\}}$ are bivariate polynomials of degree $d$. The roots of system are contained in a disc with center the origin and radius $r$. Let $\widetilde{F}=\left(\widetilde{F}_{1}, \widetilde{F}_{2}\right)$ be a $\lambda$ approximation of $F$, that is $\left\|F_{i}-\widetilde{F}_{i}\right\|_{\infty} \leq 2^{-\lambda}$. Then the zeros of $F$, $\alpha_{1}, \ldots, \alpha_{d^{2}}$, and the zeros of $\widetilde{F}, \widetilde{\alpha}_{1}, \ldots, \widetilde{\alpha}_{d^{2}}$, could be numbered such that, for $j \in[n]$,

$$
\left|\alpha_{j}-\widetilde{\alpha}_{j}\right| \leq 2^{\eta+1}
$$

where $\left.\eta=-\lambda / d^{2}+2 \tau / d^{2}+12 \lg (2 d)\right) / d+4 \lg (d) / d^{2}+\lg (r)+2$.

Proof: We consider the polynomial system $\left(S_{0}\right)$ and its resultant, $R$; after eliminating $x_{1}$ and $x_{2}$. The coefficients of $R$ are of the form $\varrho_{k} c_{1}^{d} c_{2}^{d} u^{k}$, where $0 \leq k \leq d^{2}, c_{\{1,2\}}$ denotes a monomial in the coefficients of $F_{\{1,2\}}$ of total degree $d$, and $\left|\varrho_{k}\right| \leq\left(d^{2}+2\right)^{2 d}$. The degree of $R$ wrt $u$ is $\mathcal{O}\left(d^{2}\right)$.

If we replace the polynomials $F_{\{1,2\}}$ by it approximations $\widetilde{F}_{\{1,2\}}$ and compute the resultant of the perturbed system, $\widetilde{R}$, this is also a polynomial in $u$ of degree $\mathcal{O}\left(d^{2}\right)$. Its terms are of the form $\varrho_{k} \widetilde{c}_{1}^{d} \widetilde{c}_{2}^{d} u^{k}$, where $0 \leq k \leq d^{2}, \widetilde{c}_{\{1,2\}}$ denotes a monomial in the coefficients of $\widetilde{F}_{\{1,2\}}$ of total degree $d$, and $\varrho_{k}$ is as before.

The inequality $\left\|F_{i}-\widetilde{F}_{i}\right\|_{\infty} \leq 2^{-\lambda}$ implies $\|R-\widetilde{R}\|_{\infty} \leq 2^{-\lambda+2 d \tau+12 d \lg (2 d)}$.

Let $\alpha_{j, 1}$, for $j \in\left[d^{2}\right]$, be the roots of $R$, and respectively $\widetilde{\alpha}_{j, 1}$ the roots of $\widetilde{R}$. Recall that the roots of $R$ are the $x_{1}$ coordinates of the system. Using [34, Theorem 19.1] we have the following inequality $\left|\alpha_{i, 1}-\widetilde{\alpha}_{i, 1}\right| \leq 2^{\eta}$ where $\left.\eta=-\lambda / d^{2}+2 \tau / d^{2}+12 \lg (2 d)\right) / d+4 \lg (d) / d^{2}+\lg (r)+2$.

We obtain the same bound if we replace $u-x_{1}$ with $u-x_{2}$ in $\left(S_{0}\right)$. Thus, for any root $\alpha_{j}$ of $F$ and $\widetilde{\alpha}_{i}$ of $\widetilde{F}$ we have $\left|\alpha_{i}-\widetilde{\alpha}_{i}\right| \leq 2^{\eta+1}$.

Using the previous theorem we can mimic the procedure of the univariate case. We estimate the separation bound of $\left(S_{0}\right)$ as presented in the beginning of the section. Next, we approximate $L$ to an accuracy of this order, and we obtain two approximate polynomials, and thus a perturbed system. We solve the approximate system using a numerical subdivision solver, e.g [24], and from the isolating boxes of the perturbed system we can derive isolating boxes for the roots of $\left(S_{0}\right)$ by applying Theorem 18 .

A possible alternative way of solving the bivariate polynomial systems of Problem 3 could be based on the recent work [6] on solving bivariate polynomial system of polynomials having integer coefficients.

Acknowledgments Both authors would like to thank an anonymous referee for her, or his, very detailed comments that greatly improved the presentation of the results. Elias Tsigaridas is partially supported by the French National Research Agency (ANR-09-BLAN-0371-01), GeoLMI (ANR 2011 BS03 011 06), HPAC (ANR ANR-11-BS02-013) and an FP7 Marie Curie Career Integration Grant.

\section{References}

[1] G. Alefeld and J. Herzberger. Introduction to interval computations. Academic Press, 1983.

[2] A. Baker. Linear forms in the logarithms of algebraic numbers (IV). Mathematika, 15(02):204-216, 1968 .

[3] A. Baker. The theory of linear forms in logarithms. Transcendence Theory: Advances and Applications, pages $1-27,1977$. 
[4] D. J. Bates and F. Sottile. Khovanskii-Rolle continuation for real solutions. Foundations of Computational Mathematics, 11(5):563-587, 2011.

[5] M. Bodrato and A. Zanoni. Long integers and polynomial evaluation with Estrin's scheme. In Proc. 11th Int'l Symp. on Symbolic and Numeric Algorithms for Scientific Computing (SYNASC), pages 39-46. IEEE, 2011.

[6] Y. Bouzidi, S. Lazard, G. Moroz, M. Pouget, F. Rouillier, and M. Sagraloff. Solving bivariate systems using rational univariate representations. Journal of Complexity, 37:34-75, 2016.

[7] R. Brent. Fast multiple-precision evaluation of elementary functions. J. of ACM, 23(2):242-251, 1976.

[8] R. Brent and P. Zimmermann. Modern computer arithmetic, volume 18. Cambridge University Press, 2010 .

[9] J.-S. Cheng, X.-S. Gao, and C.-K. Yap. Complete numerical isolation of real roots in zero-dimensional triangular systems. J. Symbolic Computation, 44:768-785, 2009.

[10] J. H. Davenport. Cylindrical algebraic decomposition. Technical Report 88-10, School of Mathematical Sciences, Univ. of Bath, England, available at: http://www.bath.ac.uk/masjhd/, 1988.

[11] J. Dedieu and J. Yakoubsohn. Computing the real roots of a polynomial by the exclusion algorithm. Numerical Algorithms, 4(1):1-24, 1993.

[12] D. I. Diochnos, I. Z. Emiris, and E. P. Tsigaridas. On the asymptotic and practical complexity of solving bivariate systems over the reals. J. Symbolic Computation, 44(7):818-835, 2009.

[13] Z. Du, V. Sharma, and C. K. Yap. Amortized bound for root isolation via Sturm sequences. In D. Wang and L. Zhi, editors, Int'l Workshop on Symbolic Numeric Computing (SNC), pages 113-129, Beihang University, Beijing, China, 2005. Birkhauser.

[14] A. Edelman and E. Kostlan. How may zeros of a random polynomial are real? Bulletin AMS, 32(1):1-37, 1995.

[15] A. Eigenwillig, L. Kettner, W. Krandick, K. Mehlhorn, S. Schmitt, and N. Wolpert. A Descartes Algorithm for Polynomials with Bit-Stream Coefficients. In V. Ganzha, E. Mayr, and E. Vorozhtsov, editors, CASC, volume 3718 of $L N C S$, pages 138-149. Springer, 2005.

[16] I. Z. Emiris, A. Galligo, and E. P. Tsigaridas. Random polynomials and expected complexity of bisection methods for real solving. In S. Watt, editor, Proc. 35th ACM Int'l Symp. on Symbolic \& Algebraic Comp. (ISSAC), pages 235-242, Munich, Germany, July 2010. ACM.

[17] I. Z. Emiris, B. Mourrain, and E. P. Tsigaridas. The DMM bound: Multivariate (aggregate) separation bounds. In Proc. 35th ACM Int'l Symp. on Symbolic \& Algebraic Comp. (ISSAC), pages 243-250, Munich, Germany, July 2010. ACM.

[18] D. Eppstein, M. S. Paterson, and F. F. Yao. On nearest-neighbor graphs. Discrete E Computational Geometry, 17(3):263-282, 1997.

[19] P. Escorcielo and D. Perrucci. On the davenport-mahler bound. arXiv preprint arXiv:1606.06572, 2016.

[20] M. Giusti, G. Lecerf, B. Salvy, and J.-C. Yakoubsohn. On location and approximation of clusters of zeros of analytic functions. Foundations of Computational Mathematics, 5(3):257-311, 2005. 
[21] J. Johnson and W. Krandick. Polynomial real root isolation using approximate arithmetic. In Proc. Int'l Symp. on Symb. and Algebraic Comp. (ISSAC), pages 225-232. ACM, 1997.

[22] J. R. Johnson. Algorithms for Polynomial Real Root Isolation. PhD thesis, The Ohio State University, 1991.

[23] Z. Lu, B. He, Y. Luo, and L. Pan. An algorithm of real root isolation for polynomial system. In D. Wang and L. Zhi, editors, Proc. 1st ACM Int'l Work. Symbolic Numeric Computation (SNC), pages 94-107, 2005.

[24] A. Mantzaflaris, B. Mourrain, and E. P. Tsigaridas. On continued fraction expansion of real roots of polynomial systems, complexity and condition numbers. Theoretical Computer Science, 412(22):2312$2330,2011$.

[25] J. M. McNamee and V. Y. Pan. Numerical methods for roots of polynomials (II), chapter 15. Elsevier, 2013.

[26] K. Mehlhorn and M. Sagraloff. A deterministic algorithm for isolating real roots of a real polynomial. J. Symbolic Computation, 46(1):70-90, 2011.

[27] K. Mehlhorn, M. Sagraloff, and P. Wang. From approximate factorization to root isolation with application to cylindrical algebraic decomposition. J. Symbolic Computation, 66:34 - 69, 2015.

[28] M. Mignotte. Mathematics for computer algebra. Springer-Verlag New York, Inc., 1992.

[29] M. Mignotte and M. Waldschmidt. Linear forms in two logarithms and Schneider's method. Mathematische Annalen, 231(3):241-267, 1978.

[30] V. Pan. Univariate polynomials: Nearly optimal algorithms for numerical factorization and rootfinding. J. Symbolic Computation, 33(5):701-733, 2002.

[31] V. Y. Pan and E. P. Tsigaridas. On the boolean complexity of real root refinement. In M. Kauers, editor, Proc. Int'l Symp. on Symb. and Algebraic Comp. (ISSAC), pages 299-306, Boston, USA, Jun 2013. ACM.

[32] F. Rouillier and Z. Zimmermann. Efficient isolation of polynomial's real roots. J. of Computational and Applied Mathematics, 162(1):33-50, 2004.

[33] M. Sagraloff. On the complexity of real root isolation. abs/1011.0344v1, 2010.

[34] A. Schönhage. The fundamental theorem of algebra in terms of computational complexity. Manuscript.

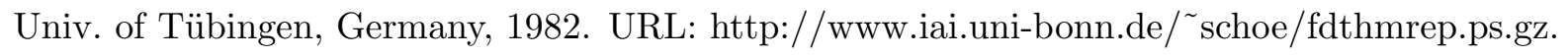

[35] A. Strzeboński and E. P. Tsigaridas. Univariate real root isolation in an extension field. In A. Leykin, editor, Proc. 36th ACM Int'l Symp. on Symbolic \& Algebraic Comp. (ISSAC), pages 321-328, San Jose, CA, USA, June 2011. ACM.

[36] A. Strzeboński and E. P. Tsigaridas. Univariate real root isolation in multiple extension fields. In Proc. 37th ACM Int'l Symp. on Symbolic \& Algebraic Comp. (ISSAC), pages 343-350, Grenoble, France, July 2012. ACM.

[37] E. P. Tsigaridas and I. Z. Emiris. On the complexity of real root isolation using Continued Fractions. Theor. Comput. Sci., 392:158-173, 2008.

[38] J. von zur Gathen and J. Gerhard. Modern computer algebra. Cambridge University Press, 3rd edition, 2013. 
[39] B. Xia and L. Yang. An algorithm for isolating the real solutions of semi-algebraic systems. J. Symbolic Computation, 34:461-477, November 2002.

[40] B. Xia and T. Zhang. Real solution isolation using interval arithmetic. Comput. Math. Appl., 52:853-860, September 2006.

[41] J. Yakoubsohn. Approximating the zeros of analytic functions by the exclusion algorithm. Numerical Algorithms, 6(1):63-88, 1994.

[42] J.-C. Yakoubsohn. Numerical analysis of a bisection-exclusion method to find zeros of univariate analytic functions. Journal of Complexity, 21(5):652-690, 2005.

[43] C. Yap. Fundamental Problems of Algorithmic Algebra. Oxford University Press, New York, 2000. 
The following is an alternative version of Lemma 2.

Lemma 19. Let $L \in \mathbb{C}$ and $\gamma_{1}$ the root of the square-free polynomial $f$ that is closest to $L$. Then

$$
|f(L)| \geq\left|a_{d}\right|^{2}\left|L-\gamma_{1}\right| 2^{-d} \mathcal{M}(f) 2^{\lg \prod_{j} \Delta_{j}} .
$$

Proof: As $\gamma_{1}$ is the root closest to $L$ it holds $\left|L-\gamma_{i}\right| \geq\left|\gamma_{1}-\gamma_{i}\right| / 2$. Then

$$
\begin{aligned}
|f(L)| & =\left|a_{d}\right| \prod_{j=1}^{d}\left|L-\gamma_{j}\right|=\left|a_{d}\right|\left|L-\gamma_{1}\right| \prod_{j \neq 1}\left|L-\gamma_{j}\right| \\
& \geq\left|a_{d}\right|\left|L-\gamma_{1}\right| \prod_{j \neq 1}\left|\gamma_{1}-\gamma_{j}\right| / 2 \\
& \geq\left|a_{d}\right|\left|L-\gamma_{1}\right| 2^{1-d} \prod_{j \neq 1}\left|\gamma_{1}-\gamma_{j}\right| \\
& \geq\left|a_{d}\right|\left|L-\gamma_{1}\right| 2^{1-d} \prod_{j \neq 1} \Delta_{j} \\
& \geq\left|a_{d}\right|\left|L-\gamma_{1}\right| 2^{1-d} \frac{1}{\Delta_{1}} \prod_{j} \Delta_{j} \\
& \geq\left|a_{d}\right|\left|L-\gamma_{1}\right| 2^{1-d} \frac{\left|a_{d}\right|}{2 \mathcal{M}(f)} 2^{\lg \prod_{j} \Delta_{j}} .
\end{aligned}
$$

For the last inequality we use $\Delta_{i} \leq 2 \mathcal{M}(f) /\left|a_{d}\right|$, that in turn relies on $\Delta_{i}=\left|\gamma_{i}-\gamma_{c_{i}}\right| \leq\left|\gamma_{i}\right|+\left|\gamma_{c_{i}}\right| \leq$ $2 \mathcal{M}(f) /\left|a_{d}\right|$. 